\title{
A acreditação e sua implementação na área de ensino pós-graduado em Saúde Pública
}

\author{
Accreditation and its implementation \\ in graduate studies in Public Health
}

Virginia Alonso Hortale 1

Monireh Obbadi 2

Célia Leitão Ramos 3

\footnotetext{
1 Departamento de Administração e Planejamento em Saúde, Escola Nacional de Saúde Pública, Fundação Oswaldo Cruz. Rua Leopoldo Bulhões 1480, Rio de Janeiro, $R J$ 21041-210, Brasil. virginia@ensp.fiocruz.br

2 Escola Nacional de Saúde Pública, Fundação Oswaldo Cruz. Rua Leopoldo Bulhões 1480, Rio de Janeiro, $R J$ 21041-210, Brasil. 3 Departamento de Ciências Sociais, Escola Nacional de Saúde Pública, Fundação Oswaldo Cruz. Rua Leopoldo Bulhões 1480, Rio de Janeiro, $R J$ 21041-210, Brasil.
}

\begin{abstract}
This article aims to contribute to a better understanding of the establishment and implementation of accreditation in graduate courses in public health and to encourage discussions to improve quality in this area. The term accreditation is defined, and two of its main thrusts are discussed: quality and competence. The basis for establishing and implementing an accreditation system is described, and some of the key characteristics of the Brazilian experience are presented.

Key words Accreditation; Quality; Teaching

Resumo O presente artigo visa contribuir para a melhor compreensão da proposta de implantação e implementação de acreditação na área de ensino pós-graduado em saúde pública, e estimular a discussão da melhoria da qualidade de seus cursos. Apresenta o significado do termo acreditação e discute dois de seus vetores constitutivos: qualidade e competência. Finalmente, discorre sobre as bases para a implantação e implementação de um sistema de acreditação, apresentando algumas características desse sistema referidos à experiência brasileira.

Palavras-chave Acreditação; Qualidade; Ensino
\end{abstract}




\section{Introdução}

Avaliações de caráter predominantemente quantitativo, levando ao estabelecimento de um ranking de excelência, têm sido insuficientes para a verificação da melhoria da qualidade da educação pretendida. Na área da formação profissional pós-graduada em saúde pública, a discussão de mecanismos que propiciem sua efetiva verificação vem acompanhada da idéia de se desenvolver sistemas de acreditação, no entanto associada, enquanto um processo complexo, a uma maior capacidade de regulação da própria sociedade. Como resultado dessa regulação, a instituição formadora tem maior visibilidade; maior transparência, com prestação de contas aos estudantes e aos profissionais da própria instituição; além de maior eficiência e eficácia de processos tanto de natureza profissional quanto educativa.

A acreditação vem sendo implementada na assistência hospitalar e na formação em saúde pública, graduada ou pós-graduada. Na primeira, as experiências focalizam a garantia da qualidade da assistência. Na segunda, focaliza a garantia da qualidade da capacitação para a prestação de serviço.

Pretendendo contribuir para a melhor compreensão dessa proposta e estimular a discussão da melhoria da qualidade da formação profissional pós-graduada na área da saúde pública, o presente artigo apresenta e discute o significado do termo acreditação e dois de seus vetores constitutivos - qualidade e competência; discorre sobre as bases para a implantação e implementação de um sistema de acreditação e apresenta algumas características retiradas da experiência brasileira de implantação desse sistema.

\section{Breve histórico}

É nos Estados Unidos da América, entre as décadas de 20 e 40, que se inicia a implementação da acreditação hospitalar e na área de formação profissional em saúde pública. Na primeira, a proposta é a de estabelecer um programa de padronização mínima da qualidade da assistência nos hospitais que são, na sua maioria, privados (O'Leary \& Donahue, 2000). Na segunda, pelo fato de existirem Escolas de Saúde Pública que titulavam profissionais com base em programas heterogêneos (tempo de duração e currículo, dentre outros), especialistas na área recomendaram que se buscasse maior uniformidade na organização desses programas. Nesse caso, é a Associação Americana de Saúde Pública (APHA) que inicia formalmente, na década de 40 , um processo de acreditação, propondo, além da uniformidade da formação, a garantia da sua qualidade, e o Conselho de Educação em Saúde Pública americana (CEPH), substituto da APHA, vem aperfeiçoando desde 1978 os critérios para acreditar as escolas de pós-graduação em saúde pública americanas.

Em 1999, a Organização Pan-Americana da Saúde divulga documento denominado Calidad y Regulación de la Educaión y del Ejercicio Profesional en Medicina (OPS, 1999), em que, além de discutir os antecedentes da crescente preocupação com a avaliação de instituições e programas de formação médica na América Latina e na região do Caribe, propõe a construção de estratégias de avaliação, incluindo os procedimentos de acreditação.

$\mathrm{Na}$ área da formação profissional em saúde pública, a prática da acreditação, embora consolidada em alguns países, não vem se dando de forma homogênea, possuindo características próprias de acordo com suas origens e referências culturais. No entanto, seu objetivo é comum, ou seja; o aperfeiçoamento de programas de formação para a qualificação de recursos humanos. Na Europa, por exemplo, esse processo se desenvolve quer devido ao baixo nível de profissionalização quer à crescente mobilidade de profissionais entre os países europeus. Tem como resultado o aumento de licenças baseadas em sistemas de verificação de qualidade da formação (Bury \& Gliber, 2001).

Atualmente, existem no Brasil, programas dedicados à avaliação do ensino da graduação. $\mathrm{Na}$ área profissional pós-graduada, embora com legislação específica, não existe obrigatoriedade de avaliações regulares, e é grande a carência de informações sobre essa formação nas diversas áreas. A partir da implantação do Sistema Único de Saúde (SUS) e do conseqüente processo de municipalização, com a alocação de profissionais de nível superior para planejamento e gerenciamento, proliferam cursos em saúde pública, grande parte deles ofertados por instituições sem tradição nesse campo.

Visando buscar alternativas para a formação de qualidade desses profissionais, a Escola Nacional de Saúde Pública da Fundação Oswaldo Cruz, juntamente com outras Escolas de Saúde Pública e Núcleos de Saúde Coletiva vêm discutindo, sob o patrocínio da Associação Brasileira de Pós-Graduação em Saúde Coletiva (ABRASCO), a implantação e implementação de um sistema de acreditação para o controle de qualidade desses cursos. É uma mudança que se inicia no plano conceitual, e depende, fundamentalmente, de educação e de 
experimentação para que novos conceitos sejam assimilados e praticados. Essa experiência iniciou-se em 1999 e pretende-se estar instalar formalmente o sistema de acreditação no Brasil em 2003.

\section{O conceito e suas características}

A palavra acreditação é pouco conhecida na língua portuguesa. São encontrados os termos acreditado (particípio de acreditar; que tem crédito; que merece ou inspira confiança; autorizado ou reconhecido por uma potência junto a outra) e acreditar (dar crédito a; crer; ter como verdadeiro; dar ou estabelecer crédito a; afiançar; conceder reputação a; tornar digno de crédito; confiança; abonar; conferir poderes a (alguém) para representar uma nação perante um país estrangeiro) (Ferreira, 1999). Na língua francesa, aparece o termo $a c$ créditer: "lui donner l'autorité nécessaire pour agir em qualité de; rendre croyable, plausible" (Rey, 1992:11). Já na língua inglesa aparece $a c$ credited: "officially recognized, generally accepted or believed, certified as being of a prescribed quality" (Cowie, 1989:9).

O termo tem como noções centrais a confiança e a qualidade, e pode ser entendido como um método de estímulo, avaliação e certificação da qualidade, além de se caracterizar como um processo educacional que introduz nas instituições a cultura da qualidade e analisa e atesta o seu grau de alcançado por elas. A acreditação deve ser diferenciada de outros processos como o licenciamento, a classificação, a habilitação ou o credenciamento pois propõe a participação voluntária, estimulando as instituições a um comportamento de procura contínua da qualidade da formação.

O conceito pode, então, ser sintetizado como uma forma de gerenciamento coletivo da qualidade, consistindo em produzir o reconhecimento social de uma determinada competência, fazendo com que a instituição formadora ou o curso se inscrevam numa perspectiva de melhoria progressiva da qualidade. A seguir, são destacados dois de seus principais vetores: - Competência - significa a "qualidade de quem é capaz de apreciar e resolver certo assunto, fazer determinada coisa, capacidade, habilidade, aptidão, idoneidade" (Ferreira, 1999:42). Pode-se caracterizar como um processo dinâmico, em que predominam práticas compartilhadas entre os atores envolvidos, que se fortalecem em um contexto de transformações tecnológicas e de formas de gestão em busca da melhoria da qualidade na produção de bens e serviços.
A abordagem da competência propõe substituir a tese da polarização das qualificações de Braverman (1972, apud Gonzalez, 1996), segundo a qual, estariam sendo criadas uma grande maioria de trabalhadores desqualificados e uma minoria de trabalhadores superqualificados. Suas principais exigências estão dadas em duas dimensões: profissional e política. A dimensão profissional leva em consideração o desenvolvimento do raciocínio lógico, as habilidades de análise de situações e habilidades estratégica e de planejamento. A dimensão política leva em consideração a possibilidade de cada indivíduo refletir e atuar criticamente sobre seu trabalho, ou seja, sua posição, função na estrutura produtiva, seus direitos e deveres.

No Brasil, a noção de competência aparece formalmente na Lei de Diretrizes e Bases da Educação Nacional (LDB) de 1996, mas ainda sem uma conceituação clara, trazendo ainda hoje, grandes desafios a serem enfrentados pelas instituições de ensino na construção de modelos de formação, necessariamente mais flexíveis e eficazes em sua estrutura.

A área da saúde, entendida enquanto um setor de prestação de serviços, apresenta algumas especificidades na sua organização e no seu processo de trabalho que justificam a discussão da idéia de competência. O setor serviços é definido como um "trabalho reflexivo" (Offe, 1989), onde o trabalhador elabora e mantém o próprio trabalho. As principais características do seu processo de trabalho podem ser assim resumidas: é heterogêneo e descontínuo, sendo difícil sua normalização técnica e a avaliação da sua produtividade. É complexo e fragmentado, decorrente da diversidade de profissões e profissionais que nele atuam, além de uma grande diversidade de usuários e tecnologias utilizadas.

Com as recentes transformações na organização do sistema de saúde brasileiro, pretende-se que o profissional seja capaz de solucionar problemas, tomar decisões e trabalhar em equipe, melhorando a qualidade dos processos, produtos e serviços prestados. Nessa perspectiva, na área da formação em saúde pública, a competência para o trabalho pode pressupor a construção de uma formação pautada na interdisciplinaridade e na história da técnica, e uma ampla base de educação geral.

- Qualidade - o emprego da idéia de qualidade é antigo. Desde o século XIX, na área industrial, foram criados tipos de controle capazes de evitar que produtos manufaturados defeituosos chegassem ao cliente, chamado de controle de qualidade. Sua expansão para outras áreas nos dá a dimensão da sua complexidade 
e do uso do seu conceito. Na área empresarial, de acordo com a International Organization for Standardization (ISO), ela significa " a totalidade de características de uma entidade que lhe confere a capacidade de satisfazer as necessidades explícitas e implícitas" (ABNT, 1994:53).

Antes considerada um fator desejável nos produtos e serviços, a qualidade passou a ser indispensável e um elemento diferenciador no processo de atendimento das expectativas de clientes e usuários. Na saúde não poderia ser de outra forma. O uso da metodologia e das ferramentas da qualidade, torna-se particularmente valioso se considerarmos a situação atual da gestão de serviços de saúde no país. Na área da atenção médica, suas definições são exploradas partindo-se do princípio de que o cuidado é um atributo que pode ter um maior ou menor grau de qualidade (Donabedian, 1980). $\mathrm{Na}$ área da educação, ressalta-se que a quantidade e a qualidade não podem ser vistas separadamente já que são complementares, e as duas são importantes para o desenvolvimento do indivíduo. "É equívoco pretender confronto dicotômico entre qualidade e quantidade. Elas não são coisas estanques, mas faceta do mesmo todo. Por mais que possamos admitir qualidade como algo 'mais' e mesmo 'melhor' que quantidade, no fundo uma jamais substitui a outra, embora sempre seja possível preferir uma à outra" (Demo, 1995:9).

A definição de quantidade tem a ver com número, volume e abundância. A qualidade, por outro lado, é uma dimensão da intensidade. “Tem a ver com profundidade, perfeição, principalmente com participação e criação. Está mais para o ser do que para o ter" (Demo, 1995:11). A qualidade é apresentada em duas dimensões: formal e política. A primeira, considera os "meios, instrumentos, formas, técnicas, procedimentos diante dos desafios do desenvolvimento". Está voltada para a "arte de descobrir. A segunda, está voltada para a competência do indivíduo de se fazer e de fazer história" (Demo, 1995:14). Está mais ligada a fins, valores e conteúdos, e à arte de fazer.

A qualidade é também uma questão de competência dos indivíduos. Precisa de consciência crítica e de capacidade de ação. Existem dois desafios: o construtivo e o participativo. A competência humana não se esgota no domínio das formas do como fazer, mas no que fazer. A qualidade é uma ação humana, porque é produzida pelo homem através da construção e da participação. É condicionada pela quantidade, mas ultrapassa-a, porque ela não é apenas acontecer, mas fazer acontecer. Assim, a educação passa a ser o espaço e o indi- cador importante da qualidade, "porque representa a estratégia básica de formação humana" (Demo, 1995:20).

Na década de 90, constatou-se que a qualidade de bens e serviços constituía-se em um fator competitivo e dependente, essencialmente, das pessoas. Para tanto, foi necessário assegurar aos egressos de cursos uma alta qualidade de ensino profissional com plena aceitação no mercado. Nesse sentido, as instituições que vêm realizando cursos na área da saúde pública, pretendem superar as concepções tradicionais associando-as ao de competência e dar corpo a um sistema de acreditação que seja ao mesmo tempo um processo de aprimoramento do desenvolvimento de recursos humanos para a saúde, conjugado com as necessidades do SUS, e de acompanhamento da qualificação das instituições de formação.

\section{Sistema de acreditação}

Um sistema de acreditação é pensado procurando atender a elementos nos planos técnico e político. Para que tenha êxito, deve fazer parte da cultura institucional e atender a interesses políticos mais gerais, e ter clareza de que suas principais definições são fruto de consenso e construído sobre as capacitações existentes e as prioridades da área.

$\mathrm{Na}$ área da formação profissional pós-graduada em saúde pública, a École Nationale de Santé Publique de Rennes (ENSP-Rennes), vem há 15 anos implementando um sistema de acreditação de programas de formação de diretores de serviços de assistência social e de hospitais, e define acreditação como o procedimento pelo qual uma instância com delegação governamental reconhece formalmente que uma instituição de ensino é competente para efetuar tarefas específicas da área, e em condições de receber o selo de qualidade. Seu sistema de acreditação é estruturado em dois níveis: técnico e político. O primeiro, é encarregado de avaliar o grau de desempenho de cada instituição em função de padrões de referência previamente estabelecidos. É composto por uma equipe de profissionais que regularmente realizam visitas de acompanhamento às instituições acreditadas ou a ser acreditadas. $\mathrm{O}$ segundo, decide sobre os encaminhamentos a serem dados.

Esses dois níveis estão presentes de forma simultânea em todo o processo, visando garantir a continuidade de uma orientação positiva de desenvolvimento e de progressão da qualidade. O acompanhamento do que foi planeja- 
do e do que foi executado não se limita à verificação de normas estabelecidas a priori, vai mais além, realizando comparações múltiplas entre aquilo que foi projetado e o que foi realizado, entre o discurso e a prática, entre os documentos e as ações empreendidas. Seu instrumento de acompanhamento se baseia em referências do ambiente externo, como por exemplo, as condições de exercício da instituição formadora, e interno, por exemplo, o projeto pedagógico e o modelo de gestão da instituição. O sistema é operacionalizado através de dois instrumentos: termo de adesão, que explicita as condições de participação da instituição no sistema e padrão de referência, que orienta a análise em diferentes dimensões.

No Brasil, vem sendo construída há três anos a proposta de um sistema de acreditação tendo como referência a experiência acima. Seus principais objetivos são: contribuir para o desenvolvimento da qualidade da formação profissional, priorizar os projetos pedagógicos voltados para o desenvolvimento do SUS e estabelecer uma regulação compartilhada entre os atores - instituições formadoras, gestores do Sistema de Saúde e sociedade - no interesse da saúde pública nacional.

Desde o início, esse processo contou com uma condução colegiada em que estão representados vários Estados e Instituições Formadoras. De setembro de 1999 até hoje, foram realizados vários encontros e seminários técnicos com instituições formadoras e de gestão do SUS. Estes objetivaram disseminar a proposta e criar consenso sobre o processo de acreditação. Alem disso, serviram para aprofundar alguns aspectos que devem ser levados em consideração na implantação de um sistema de acreditação na área da saúde pública no Brasil.

O primeiro deles, na dimensão societária, é a compreensão de que cabe à sociedade civil organizada definir os objetivos e as ações prioritárias, tendo em vista as necessidades da população. O segundo, na dimensão institucional, é de que é de responsabilidade dos educadores das instituições formadoras estimular os alunos a uma reflexão sobre suas experiências, oferecendo um conjunto de conhecimentos que sejam úteis ao seu aprimoramento. O terceiro, também na dimensão institucional, é considerar que o processo de acreditação estimula a autonomia das instituições formadoras, faz com que elas sejam incentivadas a superar obstáculos, realizando auto-avaliação permanente e, portanto, legitimando as ações que elas empreendem.

No que diz respeito ao sistema de acreditação, ele possibilita a inserção da instituição formadora em uma dinâmica de progressão con- tínua da qualidade. Também requer que a instituição proceda a uma auto-avaliação constante para alcançar a excelência de uma determinada formação pedagógica. O tempo necessário para a implantação e implementação do sistema de acreditação está diretamente relacionado ao contexto em que o processo é desencadeado.

O sistema é implantado quando todas as pessoas da instituição, desde sua direção até os profissionais do corpo administrativo que executam as tarefas mais simples, conhecerem e aplicarem no seu cotidiano as premissas que o sustentam. Para isso, as instituições devem conhecer seus processos, produtos e usuários; devem saber que a cooperação nos projetos de melhoria contínua é primordial; devem estabelecer objetivos e metas tanto em termos administrativos quanto pedagógicos; devem saber identificar claramente as relações entre causa e efeito dos processos pedagógicos pelos quais são responsáveis e, finalmente, ter indicadores de avaliação conhecidos e praticados por todos, e aprimorados com o pleno reconhecimento de suas vantagens operacionais.

\section{Considerações finais}

Entendendo que toda e qualquer proposta deve ser precedida de uma análise de seus antecedentes e limites, observa-se que muito ainda deve ser feito até que o sistema de acreditação possa ser implementado. $\mathrm{Na}$ realidade, ele pressupõe uma nova cultura institucional, que exige tempo. Na dinâmica do processo de acreditação, a avaliação é uma etapa de fundamental importância.

Tomando-se por base a experiência de outros países, considera-se que a acreditação é uma prática estratégica para controlar e garantir qualidade acadêmica e pedagógica; aperfeiçoar programas de formação voltados para o desenvolvimento do SUS; estabelecer uma regulação compartilhada entre atores acadêmicos, de serviço e da sociedade; ser possível de replicação e utilização por outras agências do Estado e, finalmente, facilitar a cooperação institucional em rede. 


\section{Referências}

ABNT (Associação Brasileira de Normas Técnicas), 1994. NBR ISO 9000 - Normas de Gestão da Qualidade e Garantia da Qualidade. Rio de Janeiro: ABNT.

BURY, A. \& GLIBER, M. (org.), 2001. Quality Improvement and Accreditation of Training Programs in Public Health. Annecy: Association of Schools of Public Health in the European Region.

COWIE, A. P. (ed.), 1989. Oxford Advanced Learner's Dictionary. Oxford: Oxford University Press.

DEMO, P., 1995. Educação e Qualidade. 2a Ed. Campinas: Papirus.

DONABEDIAN, A., 1980. Explorations in Quality: Assessment and Monitoring. v. 1. Ann Arbor: Health Administration Press.

FERREIRA, A. B. H., 1999. Novo Aurélio Século XXI. 3ạ Ed. Rio de Janeiro: Nova Fronteira.

GONZALEZ, W. R. C., 1996. Competência: Uma Alternativa Conceitual? Rio de Janeiro: Serviço Nacional de Aprendizagem Industrial/Centro Internacional para Educação, Trabalho e Transferência de Tecnologia.

OFFE, C., 1989. Trabalho \& Sociedade. Problemas Estruturais e Perspectivas para o Futuro da Sociedade do Trabalho. Rio de Janeiro: Tempo Brasileiro.

O'LEARY, D. S. \& DONAHUE, K. T., 2000 A evolução dos sistemas de acreditação de instituições de saúde. Ensaio: Avaliação de Políticas Públicas em Educação, 8:5-16.

REY, A., 1992. Le Micro-Robert. Paris: Dictionnaires Le Robert.

Recebido em 12 de julho de 2002

Aprovado em 26 de agosto de 2002 